\title{
Diagnostic potential of the diffusion tensor tractography with fractional anisotropy in the diagnosis and treatment of cervical spondylotic and posttraumatic myelopathy
}

\author{
Alessandro Landi, Gualtiero Innocenzi ${ }^{1}$, Giovanni Grasso ${ }^{4}$, Alessandro Meschini², \\ Francesco Fabbiano², Paola Castri³, Roberto Delfini \\ Department of Neurology and Psychiatry, Division of Neurosurgery, Sapienza University of Rome, Rome, Italy, Departments of ${ }^{1}$ Neurosurgery, ${ }^{2}$ Diagnostic and \\ Therapeutic Neuroradiology, INM Neuromed, Pozzilli, Italy, ${ }^{3}$ Pediatric Neurology, UTHSC (University of Tennessee Health Science Center), Memphis, Tennessee, \\ USA, ${ }^{4}$ Department of Experimental Biomedicine and Clinical Neurosciences, Neurosurgical Clinic, University of Palermo, Palermo, Italy \\ E-mail: *Alessandro Landi - dott.alessandro.landi@gmail.com; Gualtiero Innocenzi - innocenzigualtiero@tiscali.it; \\ Giovanni Grasso - giovanni.grasso@unipa.it; Alessandro Meschini - a.meschini.rad@gmail.com; Francesco Fabbiano - f.fabbiano.rad@gmail.com; \\ Paola Castri - paoliccia77@gmail.com; Roberto Delfini - delfiniroberto@gmail.com \\ *Corresponding author
}

Received: 21 June $16 \quad$ Accepted: 10 August $16 \quad$ Published: 22 September 16

\begin{abstract}
Background: Diffusion tensor imaging (DTI) is a magnetic resonance imaging (MRI)-based methodology widely used for the evaluation of microstructural integrity of the central nervous system (CNS), particularly of brain white matter fibers and bundles.

Methods: The most common parameters evaluated in a DTI study are the fractional anisotropy (FA) and mean diffusivity (MD). Combining FA and MD analyses is commonly used in the evaluation of various types of brain pathologies, such as brain tumors, where a combined analysis allows an accurate tumor characterization.

Results: Recent studies have shown that FA and MD could be of value in non-oncologic spinal pathology. In this regard, it has been demonstrated that DTI can provide new insights into the diagnosis and prognosis of cervical spondylotic myelopathy and cervical spinal cord injury.

Conclusions: Further studies are needed to assess the role of DTI in such a new clinical scenario.

Key Words: Cervical myelopathy, diffusion tensor imaging, fiber tracking, fractional anisotropy, magnetic resonance imaging, spinal cord injury
\end{abstract}

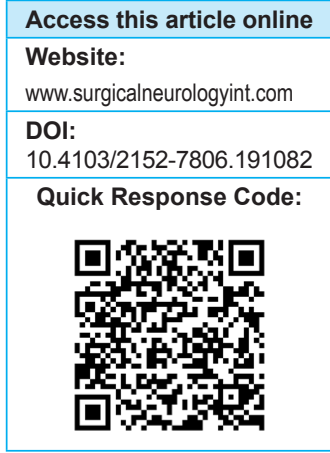

\section{INTRODUCTION}

Diffusion tensor imaging (DTI) is a magnetic resonance imaging (MRI)-based methodology widely used for the evaluation of microstructural integrity of the central nervous system (CNS), particularly of brain white matter fibers and bundles. ${ }^{[1]}$ The most common parameters that are evaluated in a DTI study are the fractional anisotropy (FA) and mean diffusivity (MD). ${ }^{[7]} \mathrm{FA}$ is a parameter that measures the tendency of water to spread in a preferred direction within a group of axons. It is a function of the axonal density and integrity of white matter fibers as well as their degree of myelination. ${ }^{[5]}$ MD is different insofar as it measures the diffusion of

This is an open access article distributed under the terms of the Creative Commons Attribution-NonCommercial-ShareAlike 3.0 License, which allows others to remix, tweak, and build upon the work non-commercially, as long as the author is credited and the new creations are licensed under the identical terms.

For reprints contact: reprints@medknow.com

How to cite this article: Landi A, Innocenzi G, Grasso G, Meschini A, Fabbiano $\mathrm{F}$, Castri P, et al. Diagnostic potential of the diffusion tensor tractography with fractional anisotropy in the diagnosis and treatment of cervical spondylotic and posttraumatic myelopathy. Surg Neurol Int 2016;7:S705-7.

http://surgicalneurologyint.com/Diagnostic-potential-of-the-diffusion-tensortractography-with-fractional-anisotropy-in-the-diagnosis-and-treatment-of-cervicalspondylotic-and-posttraumatic-myelopathy/ 
water molecules in three dimensions. A greater MD value would indicate a lower amount of cell membranes that hinder water molecule movement, as would be the case where there is a loss of axonal fibers or vasogenic edema or necrosis. Combining FA and MD analyses is commonly used in the evaluation of various types of brain and spinal oncologic pathologies, such as brain tumors, where a combined analysis enables a more accurate characterization. ${ }^{[8]}$ Recently FA and MD have been shown to be of value in non-oncologic spinal pathology. ${ }^{[5]}$ In this regard, it has been demonstrated that DTI can provide new insights into the diagnosis and prognosis of cervical spondylotic myelopathy (CSM) and cervical spinal cord injury $(\mathrm{SCI})$.

\section{CERVICAL SPONDYLOTIC MYELOPATHY}

CSM is characterized by a progressive degeneration of the cervical spinal motor unit caused by osteoarthritis or degenerative chronic compressive pathology; MRI T2 MR sequences are most commonly used to assess cord damage. Hyperintense cord signals on T2 sequences are utilized to document myelomalacia. ${ }^{[10]}$ However, the degree of spinal cord hyperintensity in T2 studies does not necessarily correlate with clinical symptoms/signs. ${ }^{[6]}$ In particular, Ellingson et al. demonstrated no association between T2 hyperintensity and neurological symptoms. ${ }^{[3]}$ They showed that FA was significantly decreased in patients with cervical spondylosis vs. healthy controls, and that the reduction in FA was higher in patients with neurological symptoms (e.g., consistent with microstructural cord damage). ${ }^{[9]}$ Another study by Cui et al. showed a reduction in FA values in patients with CSM and a strong positive correlation between FA results and the degree of stenosis. ${ }^{[2]}$ Furthermore, a recent meta-analysis compared 394 patients with CSM vs. 251 healthy controls; that study showed that in patients with CSM, the FA values were decreased whereas MD values were increased. ${ }^{[4]}$ Various microstructural conditions of the compressed cervical cord, including gliosis, microcystic degeneration, demyelination, and extracellular edema, may lead to increased MD and decreased FA. In their study, the authors found a significant decrease in FA not only in the most compressed level but also at sites distant. This finding reflects the fact that CSM-associated demyelination and axonal damage afflicted both the myelopathic lesion and the distal sites in the chronic course of the disease. Thus, the diffusion indexes from the whole cervical spinal cord could be selected to comprehensively reflect overall damage in CSM patients. Accordingly, compared with conventional MRI, DTI indexes are more sensitive in the detection of CSM patients, especially those in the early stage of myelopathy. Furthermore, quantitative analysis of DTI metrics makes it possible to evaluate severity of myelopathy and predict the outcome of surgical treatments. Figure 1 shows an



Figure I: A 67-year-old man presented with a progressive paraparesis. SagittalT2 weighted magnetic resonance images showed spinal cord compression with hyperintense cord signals at C5-C6 level (a). Diffusion tensor imaging showed decreasing in fractional anisotropy at C5-C6 level (b) but lower, at C4-C5 segment (c, d)

illustrative case.

Postoperative utility of $\mathrm{T} 2$ magnetic resonance: Fractional anisotropy and mean diffusivity analyses

Recent studies have suggested that T2 MRI could not be utilized to successfully correlate the extent of neurological damage with outcomes for patients after surgery for CSM. ${ }^{[6]} \mathrm{FA}$ and MD analyses, however, provided information regarding the structural and microstructural damage of the spinal cord that positively correlated with the extent of neurological dysfunction. ${ }^{[3,4]}$ Furthermore, in symptomatic patients with microstructural damage, FA and MD would potentially allow for more accurately assessing patients' clinical prognosis.

\section{TRAUMATIC SPINAL CORD INJURY}

Little is known regarding the potential usefulness of FA and $\mathrm{MD}$ as diagnostic and prognostic tools in assessing traumatic spinal cord injury (SCI).[11] As noted, neither clinical symptoms nor nerve fibers microstructural damage directly correlated with the altered signal revealed on T2 MRI sequences. Here, FA and MD could potentially provide useful information such as regarding the microstructural status of white fibers at the site directly affected by trauma. Furthermore, it could provide information regarding the structural integrity below and above the level injury (e.g., secondary SCI damage may extend beyond the site of the injury due to axonal degeneration). ${ }^{[1]]}$

Utility to demonstrate when and whether spinal surgery for spinal cord injury is indicated

Emergent surgical treatment (e.g., decompression) may also be indicated when SCI correlates with clinical symptoms and MRI T2 hyperintensities. For example, there may be a symptomatic patient with negative T2 
MRI findings who only requires conservative treatment. Conversely, microstructural damage highlighted by MR-documented FA and MD as well as hyperintense signals on T2 MR studies may indicate that emergency surgical decompression is warranted.

The impact of FA and MD in the evaluation of the spinal trauma is challenging. Unfortunately, these instruments, as well as the software dedicated to the elaboration of specific sequences, are not yet widely available.

\section{CONCLUSIONS}

The development of imaging techniques, such as novel MRI methods, including FA and MD sequences, provide for improved treatment options, and are especially useful in the field of complex and delicate pathologies of the spinal cord. Additional research is required to confirm the preliminary data, and multicenter prospective randomized trials will allow the clinical validation of these methods and enable their subsequent clinical application.

\section{Financial support and sponsorship Nil. \\ Conflicts of interest \\ There are no conflicts of interest.}

\section{REFERENCES}

I. Baehring JM, Fulbright RK. Diffusion-weighted MRI in neuro-oncology. CNS Oncol 2012;1:155-67.

2. Cui JL, Wen CY, Hu Y, Li TH, Luk KD. Entropy-based analysis for diffusion anisotropy mapping of healthy and myelopathic spinal cord. Neuroimage 201।;54:2I 25-31.

3. Ellingson BM, Salamon N, Hardy AJ, Holly LT. Prediction of Neurological Impairment in Cervical Spondylotic Myelopathy using a Combination of Diffusion MRI and Proton MR Spectroscopy. PLoS One 2015;10:e013945I.

4. Guan X, Fan G, Wu X, Gu G, Gu X, Zhang H, et al. Diffusion tensor imaging studies of cervical spondylotic myelopathy: A systemic review and meta-analysis. PLoS One 2015; I0:e0I I7707.

5. Landi A, Palmarini V, D'Elia A, Marotta N, Salvati M, Santoro A, et al. Magnetic resonance diffusion tensor imaging and fiber-tracking diffusion tensor tractography in the management of spinal astrocytomas. World J Clin Cases 2016;4: I-4.

6. Lin E, Long H, Li G, Lei W. Does diffusion tensor data reflect pathological changes in the spinal cord with chronic injury. Neural Regen Res 2013;8:3382-90.

7. Taoka T, Iwasaki S, Sakamoto M, Nakagawa H, Fukusumi A, Myochin K, et al. Diffusion anisotropy and diffusivity of white matter tracts within the temporal stem in Alzheimer disease: Evaluation of the "tract of interest" by diffusion tensor tractography. AJNR Am J Neuroradiol 2006;27:1040-5.

8. Wang F, Sun T, Li XG, Liu NJ. Diffusion tensor tractography of the temporal stem on the inferior limiting sulcus. J Neurosurg 2008; 108:775-78I.

9. Wang SQ, Li X, Cui JL, Li HX, Luk KD, Hu Y. Prediction of myelopathic level in cervical spondylotic myelopathy using diffusion tensor imaging. J Magn Reson Imaging 2015;41:1682-8.

10. Zhang YZ, Shen Y, Wang LF, Ding WY, Xu JX, He J. Magnetic resonance T2 image signal intensity ratio and clinical manifestation predict prognosis after surgical intervention for cervical spondylotic myelopathy. Spine 2010;35:E396-9.

II. Zhao C, Rao JS, Pei XJ, Lei JF, Wang ZJ, Yang ZY, et al. Longitudinal study on diffusion tensor imaging and diffusion tensor tractography following spinal cord contusion injury in rats. Neuroradiology 2016;58:607-14. 